\title{
Portafolio para Auto-Generación de Electricidad con Fuentes Renovables en Edificios Comerciales
}

\author{
Alvaro R. Restrepo-Garcés(1), Diego F. Manotas-Duque ${ }^{(2)}$ y Carlos A. Lozano ${ }^{(3)}$ \\ (1) Facultad de Ingeniería de Electrónica y Telecomunicaciones, Universidad del Cauca, Calle 5 No. 4-70; \\ Popayán-Colombia. (e-mail: arestrepo@unicauca.edu.co) \\ (2) Escuela de Ingeniería Industrial, Universidad del Valle, Calle 13 No. 100-00; Cali-Colombia. \\ (e-mail: diego.manotas@correounivalle.edu.co) \\ 3) Escuela de Ingeniería Eléctrica y Electrónica, Universidad del Valle, Calle 13 No. 100-00; Cali- \\ Colombia. (e-mail: carlos.a.lozano@correounivalle.edu.co)
}

Recibido Ago. 3, 2015; Aceptado Oct. 5, 2015; Versión final Nov 4, 2015, Publicado Feb. 2016

\begin{abstract}
Resumen
El objetivo de este trabajo es conformar un portafolio óptimo para la auto-generación de electricidad con fuentes de energía renovables (FER) en edificios comerciales con base en la caracterización del consumidor, la incertidumbre de las FER, la normatividad de Colombia y las opciones reales del proyecto. Se diseñó una metodología de optimización que comienza con la selección de las alternativas FER, utilizando métodos multicriterio. La evaluación económica de las alternativas se realizó con el método flujo de caja descontado. La flexibilidad del valor presente del proyecto se definió con la Teoría de Opciones Reales y el portafolio óptimo se calculó a través de la maximización del valor presente del proyecto. El caso de estudio se desarrolló en un centro comercial concluyéndose que las energías renovables son viables cuando se incluyen las opciones reales. Los retornos se incrementan con la optimización hasta en un dos mil trescientos por ciento.
\end{abstract}

Palabras clave: auto-generación; electricidad; optimización de portafolios; energías renovables; métodos multicriterio

\section{Portfolio of Self-Generation of Electricity with Renewable Sources for Commercial Buildings}

\begin{abstract}
The aim of this study is to perform an optimum electricity self-generation portfolio with renewable energy sources in commercial buildings based on consumer characterization, renewable uncertainty, Colombian regulation and the real options of the project. The optimization methodology designed starts with the selection of renewable alternatives using multi-criteria methods. The economic evaluation of the alternative options was done using the cash flow method. The flexibility of the present value of the project was defined using the Real Options theory and the optimal portfolio was estimated through maximization of the present value of the project. The case study was developed in a commercial center concluding that renewable energies are feasible when the real options are considered in the project. Returns increase with optimization up to two thousand three hundred percent.
\end{abstract}

Keywords: self-generation; electricity; portfolio optimization; renewable energy; multicriterion method 


\section{INTRODUCCIÓN}

En Colombia se promulgó la Ley 1715 de 2014 (L1715) (Congreso de la República de Colombia, 2014) que tiene por objeto: "promover el desarrollo y la utilización de las fuentes no convencionales de energía, principalmente aquellas de carácter renovable, en el sistema energético nacional, mediante su integración al mercado eléctrico..." Este marco normativo establece incentivos tributarios para promover la autogeneración en los sectores comercial, residencial e industrial; además de las posibilidades de participar en programas de respuesta a la demanda (RD) y exportar excedentes a la red (EER). Se requiere evaluar la conveniencia de implementar estas tecnologías en los sectores mencionados, para determinar las más viables desde el punto de vista técnico-económico, de acuerdo con las características del consumidor, el riesgo del proyecto y la L1715. Teniendo en cuenta que una de las maneras de gestionar el riesgo, es la adopción de un portafolio de inversión; que según Awerbuch y Yang (2008) en un portafolio de generación de energía, las FER a pesar de que son consideradas más costosas, reducirán el costo de generación y el riesgo; este trabajo tiene como objetivo principal, diseñar una metodología que permita incrementar la confiabilidad del cálculo del portafolio de un proyecto de auto-generación de electricidad (PAGE) con FER para edificios del sector comercial. De esta forma contribuir con soluciones que faciliten su valoración. La posibilidad de implementar fuentes de generación de electricidad que abastezcan las necesidades de los consumidores, que sean descentralizadas y en pequeña escala es fundamental para el desarrollo sustentable (Cervi et al., 2011); en este sentido, el sector comercial puede participar como auto-generador de electricidad, ya que son grandes consumidores, cuentan con recursos financieros para inversión e, instalaciones que en determinados casos facilitan el montaje de las tecnologías.

La metodología que se plantea se basa en el método definido por Spronk y Hallerbach (1997) para las inversiones en el sector financiero, a la cual se le realizaron una serie de ajustes: 1) caracterización del inversionista; 2) selección de las alternativas de inversión mediante métodos de toma de decisiones multicriterio (MTDM); 3) evaluación económica de los activos FER teniendo en cuenta la incertidumbre; 4) aplicación del método flujo de caja descontado (FDC), con el cual se obtienen los costos nivelados de la electricidad (CNE) y el Valor Presente Neto (VPN), los cuales junto con otros parámetros son la base para evaluar las OR del proyecto y obtener el VPN expandido (VAf) y; 5) optimización del portafolio con procesos de simulación de las variables.

Entre los método MTDM, según Taha y Daim (2013), el Proceso Analítico Jerárquico (AHP, en inglés) es el de mayor aplicación en el sector eléctrico. El AHP ha sido aplicado entre otros, por: San Cristobal (2011) para seleccionar las mejores tecnologías FER, en el marco del Plan de Energías Renovables del gobierno Español y; Aragonés et al., (2014) determinan la prioridad de inversión en tecnologías fotovoltaicas (FV). Algunos investigadores con el ánimo de darle mayor robustez a los resultados, combinan el uso de AHP con diferentes métodos, por ej: Loken y Botterud (2005) aplican la Teoría de Utilidad Multi-atributo y AHP para la planeación de un sistema local de energía; Ceciliano et al., (2007) utilizan AHP y programación lineal multiobjetivo y lo aplican a un problema de expansión de la generación; Wang et al., (2008) seleccionan un sistema de trigeneración a instalar en un edificio, para lo cual utilizan AHP y la Técnica para Ordenar Preferencias Mediante Similitud a la Solución Ideal (TOPSIS, en inglés) y; Alcan et al., (2013) utilizan AHP y TOPSIS con el fin de seleccionar el mejor sistema de cogeneración para un hotel. Siguiendo la estrategia de utilizar un método que complemente el AHP para reducir sus debilidades, en este trabajo se desarrolló un método que combina AHP y TOPSIS para la selección de las alternativas de generación de un PAGE.

La TOR es utilizada para determinar el VAf, ésta se fundamenta en la teoría de opciones financieras, añadiendo la capacidad de modelar explícitamente los efectos de las fuentes de incertidumbre individuales y contabilizando la flexibilidad que los administradores de proyectos tienen en cuenta a menudo sobre el momento de inversión frente a los flujos de efectivos futuros. Algunos trabajos que han considerado las OR, son: Sezgen y Krishnarao (2007) presentan varios modelos de OR para diferentes tipos de acciones de RD; Kienzle et al., (2012) desarrollan un modelo "Hub", que describe la conversión y almacenaje de múltiples despachadores de energía, la evaluación económica del modelo la hacen utilizando Simulación de Monte Carlo (SMC) y TOR; Martinez et al., (2012 ) valora un proyecto FV y las características de un programa RD para una residencia desconectada de la red de servicios. Los RD se centran en la conformación y la nivelación de la carga eléctrica por parte del consumidor; de acuerdo con el Departamento de Energía de E.U (2006) son una vía para lograr mejoras en la eficiencia energética. La aplicación de TOR incluyendo programas RD es escaza, y debido a la no consideración de este tipo de programas en Colombia antes de la L1715, no se conocen trabajos previos en el contexto del mercado colombiano. Los programas RD en este proyecto, son considerados como una opción real que tiene el inversor sobre la gestión del proyecto y una oportunidad para mejorar la valoración de los portafolios. 
Modelos de optimización de portafolio han sido desarrollados entre otros, por: Delarue et al., (2011) que consideran la optimización del portafolio desde un punto de vista social y se enfocan en hacer la distinción entre energía y potencia; Bhattacharya y Kojima (2012) modelan la oferta de electricidad de un generador a través de la minimización los riesgos de inversión en el portafolio; Fuss et al., (2012) desarrollan un modelo basado en TOR, para analizar el impacto de la incertidumbre en la toma de decisiones a nivel de planta, utilizan una base de datos como punto de partida para establecer el portafolio de tecnologías óptimas en diferentes escenarios socio-económicos; enfocándose en particular en las bajas emisiones; Manotas et al., (2014) determinan la cantidad óptima de energía a suministrar a los consumidores eléctricos teniendo en cuenta las opciones presentes en el mercado eléctrico colombiano; Belderbos y Delarue (2015) definen un portafolio FER a través de la optimización de los costos en las etapas de planeación y operación de un sistema de generación, teniendo en cuenta restricciones técnicas y la demanda, con resolución temporal de una hora y horizonte de tiempo de un año.

De acuerdo con las revisiones de la literatura, no se encuentran trabajos que presenten un portafolio de PAGE para consumidores comerciales, considerando un método integral de optimización y las opciones implícitas en el marco regulatorio de un país, por lo tanto este trabajo es una aporte para el sector energético y contribuye con una metodología que incorpora técnicas de diferentes áreas de conocimiento en un sólo método de trabajo. La aplicación de la metodología se realiza a través de un caso de estudio para un Centro Comercial (CC), en una ciudad colombiana. Los resultados del trabajo indican: 1) los incentivos tributarios de la L1715 y las OR hacen viable los PAGE en los escenarios considerados en este trabajo; 2) las FV contribuyen en mayor grado al portafolio, y la biomasa no es viable técnicamente para los CC; 3 ) la optimización del portafolio a través de la maximización del VA $A_{f}$, mejora los retornos del proyecto y; 4) el modelo es aplicable a otros sectores y en otros países bajo las condiciones específicas de cada caso.

\section{METOdOLOGÍA}

El método propuesto para la optimización de un portafolio PAGE se presenta en la Fig 1. A continuación se hace la descripción de cada una de las etapas.

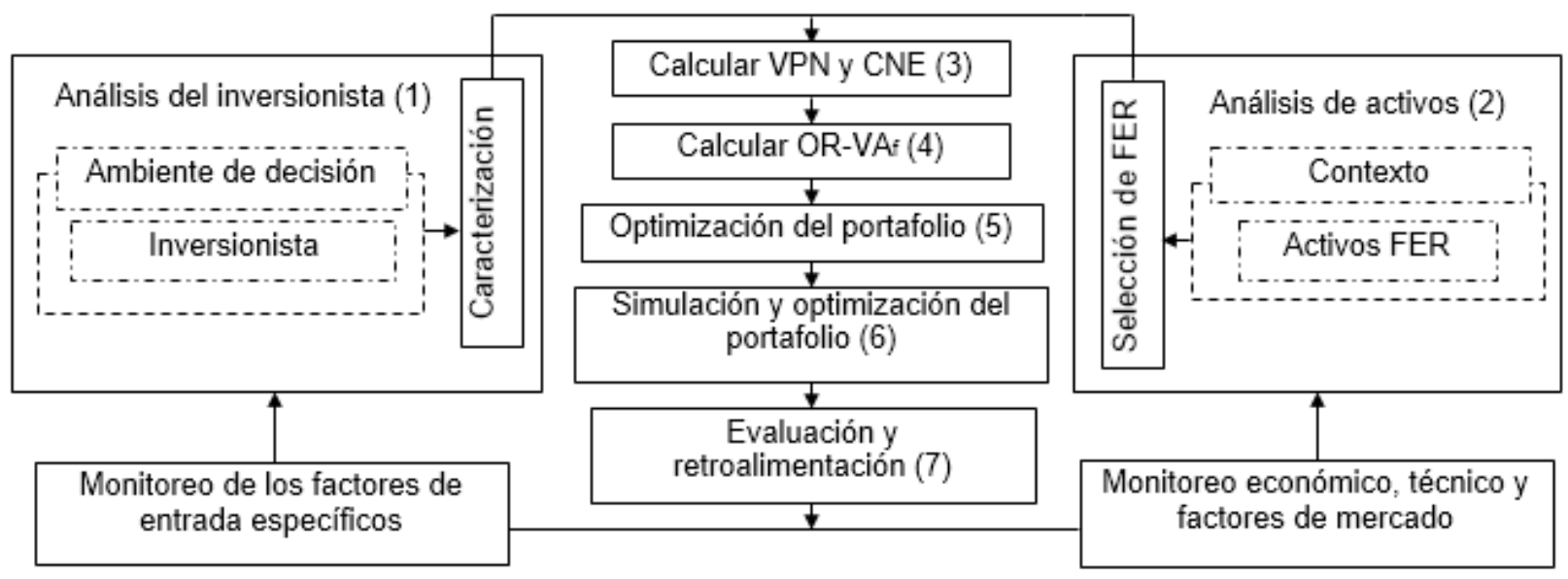

Fig. 1: Metodología de formación de un portafolio optimizado de FER, adaptado de Spronk y Hallerbach (1997)

Etapa 1. El objetivo es definir el perfil de carga y usos de la electricidad del consumidor (inversionista). La caracterización debe reflejar el contexto de decisión, los objetivos que se desean atender, las restricciones y preferencias. La caracterización es un factor clave en el proceso de toma de decisiones.

Etapa 2. Según Stirling (2010) "es necesario analizar los atributos de cada cesta en que se pondrán los huevos"; con este fin, se propone un proceso (ver Fig 2) de selección de las alternativas $\left(A_{j}\right)$ FER a través de AHP-TOPSIS y considerando la incertidumbre de los criterios $\left(C_{i}\right)$ a través de procesos de SMC.

Etapa 3. Se calcula el valor presente neto promedio del portafolio $\left(\overline{\mathrm{VPN}}_{\mathrm{p}}\right)$ y los costos nivelados promedio de la electricidad ( $\overline{\mathrm{CNE}}$ ) con las ecs.(1) y (2), respectivamente; y considerando los beneficios de la $\mathrm{L} 1715$, Según Lee (2011), el FDC no incluye la incertidumbre de las FER, por lo cual su eficacia es objetable; para contrarrestar esa deficiencia, se simulan las variables estocásticas y el FDC (ver Fig 3.). 

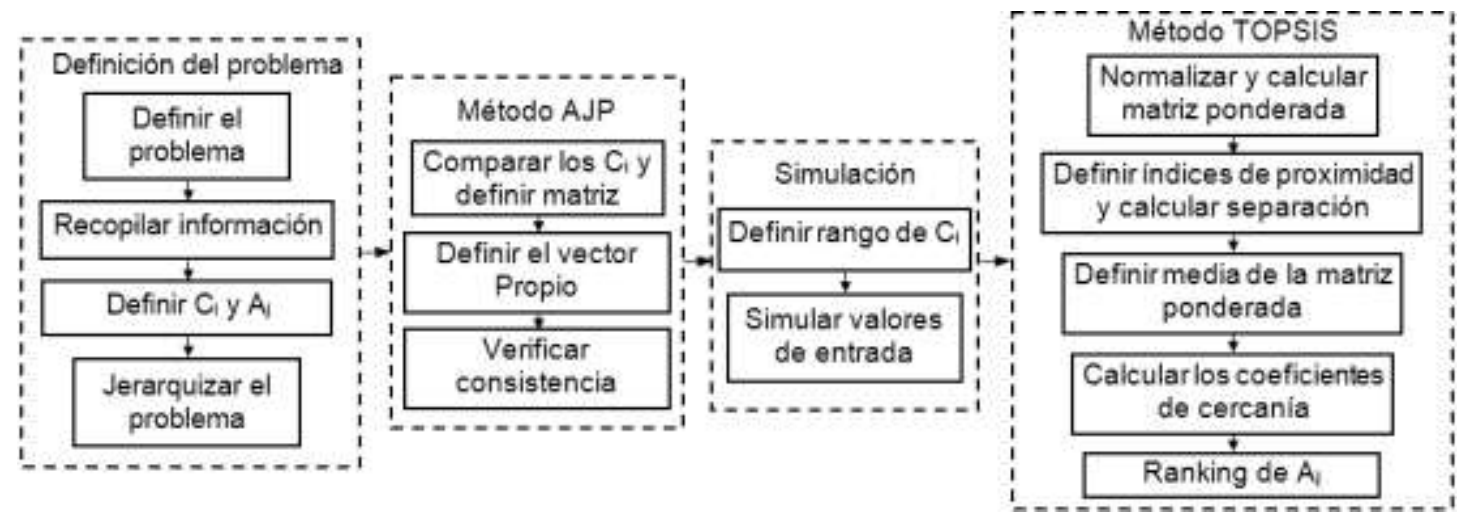

Fig. 2. Proceso de selección de las alternativas FER

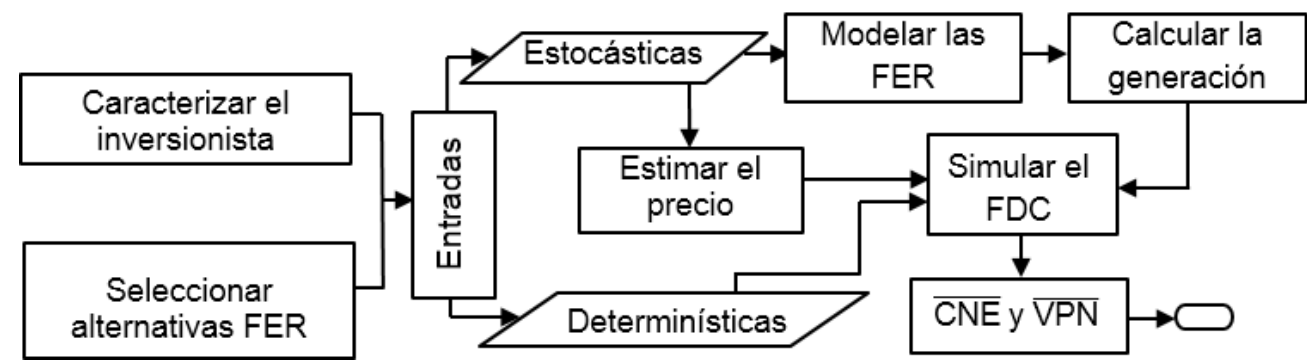

Fig. 3. Proceso para la evaluación económica de un proyecto de auto-generación FER

$\left.\overline{\operatorname{VPN}}_{p}=\left[\sum_{1}^{t} \frac{\left(B_{i}+\bar{B}_{g p}\right)}{(1+W A C C)^{t}}-\frac{C_{p}}{(1+W A C C)^{t}}\right]-I_{0}-A D-E D\right)$

$\overline{\mathrm{CNE}}=\left(\mathrm{I}_{\mathrm{o}}+\mathrm{C}_{\mathrm{p}}-\mathrm{B}_{\mathrm{i}}\right) / \overline{\mathrm{G}}$

Donde $B_{i}$ es el beneficio por deducción del $50 \%$ de la renta sobre las inversiones iniciales $\left(I_{0}\right)$, en un plazo máximo de cinco años; $\bar{B}_{g p}$ es el valor promedio de los beneficios resultado de la gestión del portafolio; $C_{p}$ son los costos totales del portafolio; WACC es la tasa de descuento que mide el costo del capital; $t$ es el número de periodos; $A D$ es la deducción del Impuesto al Valor Agregado sobre $I_{0}$, ED exención de aranceles por importaciones y, $\overline{\mathrm{G}}$ es la generación promedio total del sistema. Todos los valores son calculados en el año cero. Los $\bar{B}_{g p}$ de generación FER, se pueden presentar por: sustitución de compras a la red de servicios $\left(\bar{B}_{\mathrm{sp}}\right)$ y por exportación de excedentes a la red $\left(\bar{B}_{\mathrm{ep}}\right)$ :

Sustitución de compras a la red de servicios de un portafolio $\left(\overline{\mathrm{B}}_{\mathrm{sp}}\right)$. En el caso que el precio de compra del kWh (PC) por parte del cliente eléctrico se mantenga constante en todo el periodo, se calculan con:

$\overline{\mathrm{B}}_{\mathrm{sp}}=\sum_{1}^{\mathrm{t}} \operatorname{Min}\left\{\left(\overline{\mathrm{G}}_{1}+\overline{\mathrm{G}}_{2} \cdots,+\overline{\mathrm{G}}_{\mathrm{n}}\right)^{*} \overline{\mathrm{PC}} ;\left(\mathrm{D}_{\mathrm{T}}^{*} \overline{\mathrm{PC}}\right)\right\}$

Si el portafolio FER se encuentra inmerso en un mercado en que existe diferenciación de tarifas entre los periodos de demanda pico $\left(D_{p}\right)$ y valle $\left(D_{v}\right)$, la ecuación anterior se transforma en:

$\overline{\mathrm{B}}_{\mathrm{sp}}=\sum_{1}^{\mathrm{t}} \operatorname{Min}\left\{\left(\overline{\mathrm{G}}_{1 \mathrm{v}}+\overline{\mathrm{G}}_{2 \mathrm{v}} \cdots,+\overline{\mathrm{G}}_{\mathrm{nv}}\right)^{*} \overline{\mathrm{T}}_{\mathrm{v}}+\left(\overline{\mathrm{G}}_{1 \mathrm{p}}+\overline{\mathrm{G}}_{2 \mathrm{p}} \cdots,+\overline{\mathrm{G}}_{\mathrm{np}}\right)^{*} \overline{\mathrm{T}}_{\mathrm{p}} ;\left(\mathrm{D}_{\mathrm{v}}{ }^{*} \overline{\mathrm{T}}_{\mathrm{v}}+\mathrm{D}_{\mathrm{p}}{ }^{*} \overline{\mathrm{T}}_{\mathrm{p}}\right)\right\}$

Exportación de excedentes a la red $\left(\bar{B}_{e p}\right)$, se calculan con: 
$\overline{\mathrm{B}}_{\mathrm{ep}}=\sum_{1}^{\mathrm{t}} \operatorname{Max}\left\{\left(\overline{\mathrm{G}}_{\mathrm{e} 1}+\overline{\mathrm{G}}_{\mathrm{e} 2} \cdots,+\overline{\mathrm{G}}_{\mathrm{en}}{ }^{*} \overline{\mathrm{T}}_{\mathrm{e}}\right) ; 0\right\}$

Donde $\overline{P C}$ es el precio promedio de compra de un kWh que se estimó con una red neuronal artificial al cual se le adicionan los subsidios y aportes establecidos en la normatividad colombiana; $\overline{\mathrm{G}}_{\mathrm{n}}$ son los kWh promedio generados por cada uno de los activos FER, $D_{T}$ es la demanda total del consumidor; $\overline{\mathrm{T}}_{\mathrm{v}}, \overline{\mathrm{T}}_{\mathrm{p}}$ y $\overline{\mathrm{T}}_{\mathrm{e}}$ son las tarifas promedio del kWh: valle, pico y de exportación, respectivamente y, $\overline{\mathrm{G}}_{\mathrm{en}}$ son excedentes de generación promedio de cada activo del portafolio.

Etapa 4. La L1715 plantea por primera vez a los consumidores la posibilidad de exportar excedentes a la red (EER) y participar en programas RD; son considerados en este trabajo como opciones reales y se denominan, respectivamente $\mathrm{OR}_{\mathrm{e}}$ y $\mathrm{OR}_{\mathrm{RD}}$. Se conforma arbitrariamente un portafolio con las alternativas seleccionadas en el paso 2 y, se aplica TOR empleando el método del árbol binomial (MB) (ver Fig 4) con valoración neutral al riesgo (Cox, Ross, y Rubinstein, 1979), el cual ha sido aplicado entre otros, por Santos, et al., (2014). En este trabajo se supuso para las dos opciones, un incremento de las tecnologías a instalar, por esta razón se consideran como una OR de ampliación, cuyo cálculo se realiza de acuerdo con el siguiente planteamiento.

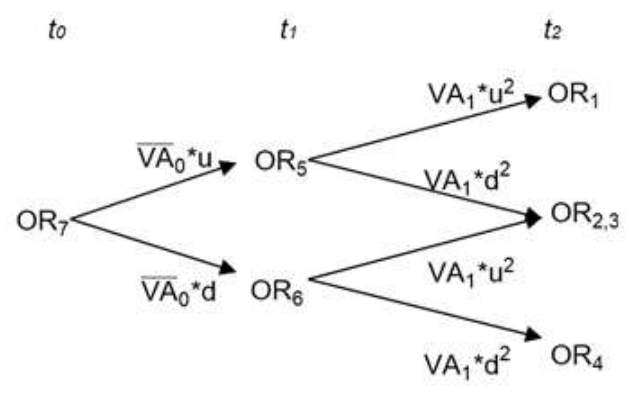

Fig. 4. Árbol Binomial para el cálculo de la $\mathrm{OR}_{\mathrm{e}} \mathrm{u} \mathrm{OR}_{\mathrm{RD}}$

Para el cálculo de las OR con el MB se siguen dos fases:

Fase 1. Se determina el valor de $\mathrm{OR}_{\mathrm{e}} \mathrm{u} \mathrm{OR}_{\mathrm{RD}}$ en cada uno de los nodos terminales, siguiendo el proceso indicado en el árbol (ver

Fig. 4) y aplicando las ecuaciones (6) y (7).

$$
\begin{aligned}
& \mathrm{OR}_{1}=\overline{\mathrm{VA}}_{0} \mathrm{u}^{\mathrm{t}}+\max \left[\left(\overline{\mathrm{B}}_{\mathrm{gp}}+\overline{\mathrm{VA}}_{0}\right)^{*} \mathrm{u}^{\mathrm{t}}-\mathrm{K}^{\prime} ; 0\right] ; \quad \mathrm{OR}_{2,3}=\overline{\mathrm{VA}}_{0} \mathrm{u}^{\mathrm{t}} \mathrm{d}^{\mathrm{t}}+\max \left[\left(\overline{\mathrm{B}}_{\mathrm{gp}}+\overline{\mathrm{VA}}_{0}\right)^{*} \mathrm{u}^{\mathrm{t}} \mathrm{d}^{\mathrm{t}}-\mathrm{K}^{\prime}, 0\right] \quad \mathrm{y} \\
& \mathrm{OR}_{4}=\overline{\mathrm{VA}}_{0}{ }^{*} \mathrm{~d}^{\mathrm{t}}+\max \left[\left(\overline{\mathrm{B}}_{\mathrm{gp}}+\overline{\mathrm{VA}}_{0}\right)^{*} \mathrm{~d}^{\mathrm{t}}-\mathrm{K}^{\prime} ; 0\right] \\
& \mathrm{u}=\mathrm{e}^{\sigma} ; \mathrm{d}=1 / \mathrm{u}
\end{aligned}
$$

Donde $\overline{\mathrm{VA}}_{0}$ es el valor actual medio del proyecto sin EER o sin RD (escenario base) en el momento cero; $\bar{B}_{g p}$ son los beneficios de gestión del portafolio con EER y/o RD; $t$ es el periodo o periodos en que se pueden ejercer las OR; $\mathrm{K}^{\prime}$ los costos causados por la ampliación del portafolio; u y d son los factores de ascenso y descenso y, $\sigma$ es la volatilidad el proyecto que se calculó de acuerdo con el método "market asset disclaimer" propuesto por Copeland (2001).

Fase 2. En esta etapa se lleva a cabo un proceso de inducción regresiva, de acuerdo con:

$$
\begin{aligned}
& \mathrm{OR}_{5}=\left[\frac{\left(\mathrm{OR}_{1}{ }^{*} \mathrm{p}\right)+\left(\mathrm{OR}_{2}{ }^{*}(1-\mathrm{p})\right.}{1+W A C C}\right] ; \mathrm{OR}_{6}=\left[\frac{\left(\mathrm{OR}_{2}{ }^{*} \mathrm{p}\right)+\left(\mathrm{OR}_{3}{ }^{*}(1-\mathrm{p})\right.}{1+W A C C}\right] \text { y } \mathrm{OR}_{7}=\left[\frac{\left(\mathrm{OR}_{5}{ }^{*} \mathrm{p}\right)+\left(\mathrm{OR}_{6}{ }^{*}(1-\mathrm{p})\right.}{1+W A C C}\right] \\
& \mathrm{p}=\left(1+\mathrm{r}_{\mathrm{f}}\right)-\mathrm{d} / \mathrm{u-d} ; \mathrm{q}=1-\mathrm{p}
\end{aligned}
$$

Donde $(p)$ es la probabilidad de un incremento y $(q)$ de un decremento $y, r_{f}$ es la tasa libre de riesgo. 
El valor del proyecto en $\mathrm{t}=0\left(\mathrm{OR}_{7}\right.$, en la Fig 4$)$, se calcula con:

$\mathrm{VA}_{\mathrm{f}}=\overline{\mathrm{VPN}}_{\text {basic }}+\mathrm{OR}_{7}$

Donde $\mathrm{VA}_{\mathrm{f}}$ es el valor del proyecto con flexibilidad en el año cero y $\overline{\mathrm{VPN}}_{\text {basic }}$ es el valor promedio del proyecto sin flexibilidad (sin opciones).

La $\mathrm{OR}_{\mathrm{RD}}$ se calculó de acuerdo con el "método momentos de uso" (TOU, en inglés), que de acuerdo con el Departamento de Energía de E.U. (2006), consiste en que el precio del kWh varía de manera predeterminada dentro de ciertos periodos de tiempo (ej: horas pico y valle, semana y fines de semana, etc). Se suponen dos tipos de tarifas: en periodo valle $\left(T_{v}\right)$ y en periodo pico $\left(T_{p}\right)$; se asume que:

$\mathrm{T}_{\mathrm{v}}=\overline{\mathrm{PC}}$

$\mathrm{T}_{\mathrm{p}}=\overline{\mathrm{PC}}^{*}(1+\mathrm{s})$

Donde s es un porcentaje de recargo en el periodo pico.

El modelo de optimización de RD le da prioridad a la $D_{p}$, porque en los programas TOU el costo del kWh es mayor en los periodos pico. La gestión del portafolio con programas RD se determina con:

$$
\begin{aligned}
& \overline{\mathrm{B}}_{\mathrm{pp}}=\operatorname{Min}\left\{\left[\left(\overline{\mathrm{G}}_{1 \mathrm{p}}+\overline{\mathrm{G}}_{2 \mathrm{p}} \cdots,+\overline{\mathrm{G}}_{\mathrm{np}}\right){ }^{*} \overline{\mathrm{T}}_{\mathrm{p}} ; \mathrm{D}_{\mathrm{p}}{ }^{*} \overline{\mathrm{T}}_{\mathrm{p}}\right]\right\} \\
& \overline{\mathrm{B}}_{\mathrm{pv}}=\operatorname{Max}\left\{\operatorname{Min}\left[\left(\overline{\mathrm{G}}_{1 \mathrm{v}}+\overline{\mathrm{G}}_{2 \mathrm{v}} \cdots,+\overline{\mathrm{G}}_{\mathrm{nv}}-\mathrm{D}_{\mathrm{p}}\right) * \overline{\mathrm{T}}_{\mathrm{v}} ; \mathrm{D}_{\mathrm{v}}{ }^{*} \overline{\mathrm{T}}_{\mathrm{v}}\right] ; 0\right\} \\
& \overline{\mathrm{B}}_{\mathrm{RDp}}=\overline{\mathrm{B}}_{\mathrm{pp}}+\overline{\mathrm{B}}_{\mathrm{pv}}
\end{aligned}
$$

Donde $\bar{B}_{p p}$ y $\bar{B}_{p v}$ son respectivamente los beneficios promedio del portafolio en los periodos pico y valle; $\bar{B}_{R D p}$ es el valor medio de los beneficios del portafolio obtenidos de los programas $R D$. $\bar{B}_{R D p}$ se sustituye en la ecuación (1) en lugar de $\bar{B}_{g p}$ para determinar el VPN del proyecto y en (6) para determinar el VA $A_{f}$.

Etapa 5. Se plantean dos modelos de optimización [ver ecuaciones (14) y (15)] similares en su planteamiento pero diferentes en las variables y OR de base. Los dos buscan la maximización del $V A_{f}$ del portafolio que incluye las $\mathrm{OR}_{\mathrm{e}}$ y $\mathrm{OR}_{\mathrm{RD}}$. Se consideran como restricciones la capacidad de instalación (es el número de equipos que se pueden instalar, acorde al área total disponible) y la demanda del edificio comercial.

El modelo parte de la definición de un escenario base $(E B)$ que cuenta con una capacidad instalada $\left(C_{n}^{\text {actual }}\right)$ y una capacidad límite $\left(C_{n}^{\max }\right)$, y busca maximizar el $V_{f}$. En el caso de $\mathrm{OR}_{\mathrm{e}}$, el modelo de optimización requiere que la sumatoria de $\bar{G}_{n}$ del portafolio, sea mayor a $D_{v}$; de esta manera, se cubren las necesidades del edificio comercial.

El modelo de optimización $\mathrm{OR}_{\mathrm{RD}}$, además de lo ya comentado, necesita que la generación del portafolio FER sea mayor que la demanda pico $\left(D_{p}\right)$; de esta forma se atiende en primer lugar el periodo con tarifas más altas y posteriormente el de tarifas bajas. Si se presentan excedentes, éstos son vendidos a la red. La Tabla 1 resume las ecuaciones que describen los modelos.

Etapa 6. Se realiza el proceso de simulación y optimización combinados para permitir la optimización de los modelos con factores inciertos. El software RiskOptimizer 5.5 es la herramienta empleada. El optimizador usa los resultados de ejecuciones sucesivas del modelo de simulación para guiar su búsqueda de mejores soluciones y óptimas.

Etapa 7. Se analizan los resultados, se realizan ajustes y se determina el mejor portafolio para el proyecto. 
Tabla 1: Modelos de optimización del $\mathrm{VA}_{\mathrm{f}}$

\begin{tabular}{|c|c|c|c|}
\hline Optimización $\mathrm{OR}_{\mathrm{e}}$ & & Optimización $\mathrm{OR}_{\mathrm{R}}$ & \\
\hline $\operatorname{Max}: \overline{\mathrm{VA}}_{\mathrm{f} 1}$ & (14) & $\operatorname{Max}: \overline{\mathrm{VA}}_{\mathrm{f} 2}$ & (15) \\
\hline Sujeto a: & & Sujeto a: & \\
\hline $\mathrm{C}_{1}^{\text {actual }} \leq \mathrm{C}_{1} \leq \mathrm{C}_{1}^{\max }$ & & $\mathrm{C}_{1}^{\text {actual }} \leq \mathrm{C}_{1} \leq \mathrm{C}_{1}^{\max }$ & \\
\hline $\mathrm{C}_{2}^{\text {actual }} \leq \mathrm{C}_{2} \leq \mathrm{C}_{2}^{\max }$ & & $\mathrm{C}_{2}^{\text {actual }} \leq \mathrm{C}_{2} \leq \mathrm{C}_{2}^{\max }$ & \\
\hline $\mathrm{C}_{\mathrm{n}}^{\text {actual }} \leq \mathrm{C}_{\mathrm{n}} \leq \mathrm{C}_{\mathrm{n}}^{\max }$ & & $\mathrm{C}_{\mathrm{n}}^{\text {actual }} \leq \mathrm{C}_{\mathrm{n}} \leq \mathrm{C}_{\mathrm{n}}^{\max }$ & \\
\hline$\sum_{1}^{n} \bar{G}_{n}>D_{v}$ & & $\sum_{1}^{n} \bar{G}_{n}>D_{p}$ & \\
\hline
\end{tabular}

La metodología planteada se aplicó a los centros comerciales (CCs), considerando que sus edificios son lugares con características espaciales y arquitectónicas que brindan mayores posibilidades para la implementación de tecnologías FER. En el caso de estudio solo se tuvo en cuenta la demanda de electricidad que está a cargo de la administración del CC (oficinas y áreas comunes). Se analizaron sesenta y nueve CCs en Colombia (36,3\% del total), según la información obtenida, los índices promedio, son: número de locales 200, área construida $91.412,70 \mathrm{~m}^{2}$ y número de parqueaderos de automóviles 1.024. De acuerdo con estudios realizados en Colombia por UPME-CORPOEMA (2013), el uso de electricidad en los CC de mediano tamaño es: iluminación $(43,6 \%)$, acondicionamiento de espacios $(33,1 \%)$, refrigeración $(12,6 \%)$, fuerza motriz $(4,8 \%)$, equipos de oficina $(4.3 \%) y$, otros $(1,5 \%)$

La demanda de potencia y los precios de la electricidad se determinaron con base en la información provista por una empresa de servicios que vende electricidad a cuatro CCs de mediano tamaño. En la Fig 5 se aprecia la demanda durante los doce meses del año 2013, se observan pequeños incrementos de octubre a noviembre; y la Fig 6 presenta la demanda promedio anual por hora del año 2013, el consumo se incrementa desde las 17:00 hasta las 22:00, el resto del periodo no presenta cambios abruptos. El promedio total de los precios de compra de los cuatro CCs de referencia para el año 2013 fue de 15,06 \& de dólar U.S./kWh con una desviación de 1,59 \$/kWh, precio superior al promedio de $\mathrm{E}$.U en el 2012 para el sector comercial, que según la Agencia Internacional de Energía (2014) fue de 10,12 \$/kWh. Con base en la información presentada se diseñó el escenario base, el cual sirvió de referencia para el proceso de selección de las alternativas.

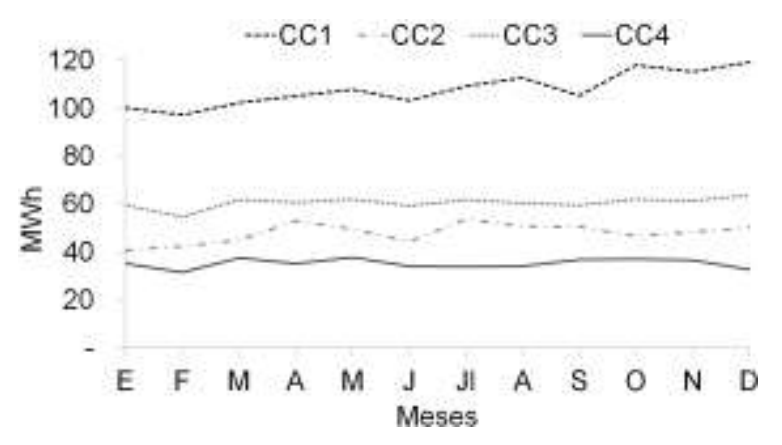

Fig. 5. Demanda mensual de electricidad

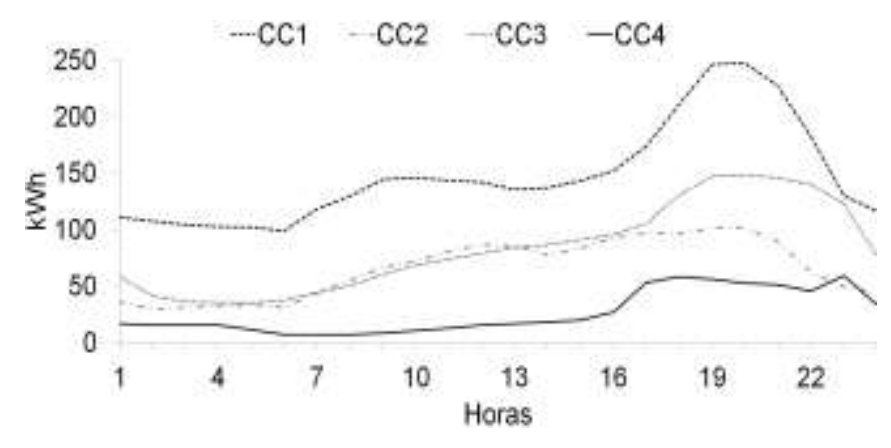

Fig. 6. Demanda horaria promedio anual

\section{RESULTADOS}

Selección de las alternativas. La Fig 7 presenta el árbol jerárquico del problema, los criterios $\left(\mathrm{C}_{\mathrm{i}}\right)$ y las alternativas fueron definidos a través de dos procesos: 1) revisión de la literatura de lbáñez y Perez, (2014) y Jiang, et al.,(2009), quienes respectivamente, realizan una clasificación de los criterios aplicados en la industria y en el sector de las renovables, y 2) consulta de siete expertos en el tema. La calificación de los criterios se realizó por parte de diez expertos y, la determinación del vector propio $(\vec{w})$ y el cálculo del Ratio de Consistencia (RC) se hizo de acuerdo con Saaty y Vargas, (2001). Los resultados se presentan en la Tabla 1 , siendo $\mathrm{C}_{2}$ el de mayor valor, seguido de $\mathrm{C}_{5} \mathrm{y}, \mathrm{C}_{1}$ el de menor. 


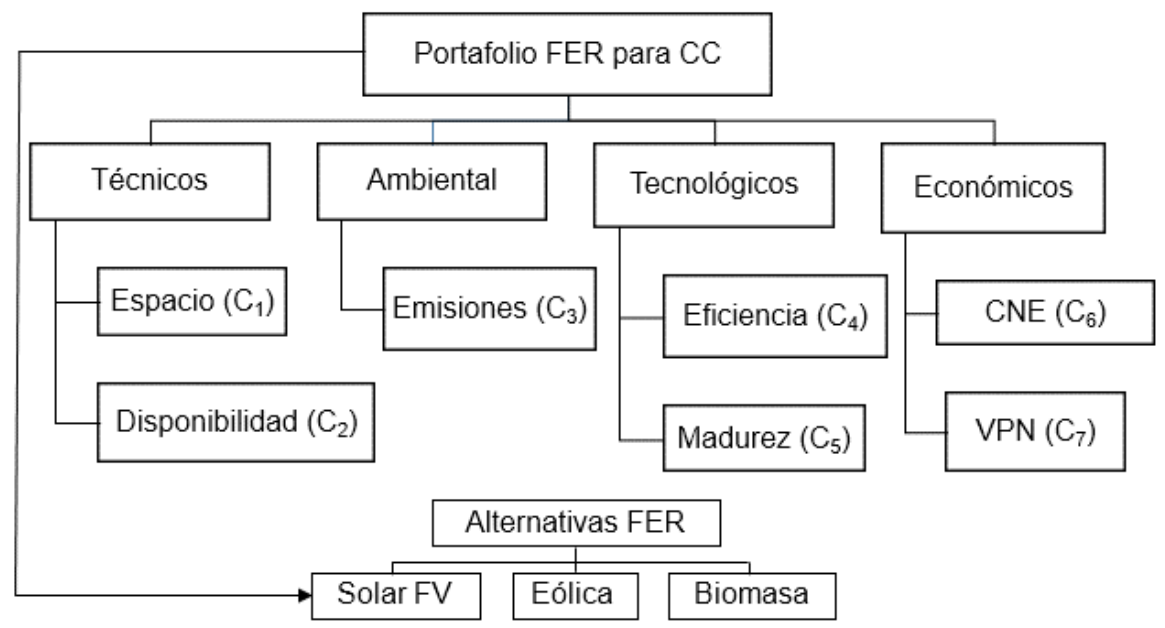

Fig. 7. Árbol jerárquico del problema

Tabla 1. Peso de los criterios, con base en juicios de los expertos.

\begin{tabular}{|c|c|c|c|c|c|c|c|c|}
\hline & $\mathrm{C}_{1}$ & $\mathrm{C}_{2}$ & $\mathrm{C}_{3}$ & $\mathrm{C}_{4}$ & $\mathrm{C}_{5}$ & $\mathrm{C}_{6}$ & $\mathrm{C}_{7}$ & $\mathrm{RC}$ \\
\hline $\overrightarrow{\mathrm{w}}$ & 0,06 & 0,32 & 0,10 & 0,09 & 0,15 & 0,11 & 0,17 & 0,0321 \\
\hline
\end{tabular}

Para el cálculo de los rangos de los criterios $\left(\mathrm{C}_{2}\right),\left(\mathrm{C}_{6}\right)$ y $\left(\mathrm{C}_{7}\right)$, a partir de la información de los cuatro CCs, se definió un escenario base (EB) con los valores promedio de los CCs. El EB tiene las siguientes características: área construida, $41.000 \mathrm{~m}^{2}$; número de locales, 150; número de parqueaderos, 460 (tamaño de cada parqueadero 2,3 × 2,5 m) y área total parqueaderos, $3.703 \mathrm{~m}^{2}$ (se incluyen vías de circulación). La demanda promedio en kWh del CC, es: total $\left(D_{T}\right)$ 3.033/día; pico/día $\left(D_{p}\right) 1.130,77$ y; valle/día $\left(D_{v}\right)$ 1902,63. Se supuso la ubicación del CC en la ciudad de Barranquilla (Lat: 10,9638 y Long: $-74,796389^{\circ}$ y Altitud: $0 \mathrm{msnm}$ ).

La Tabla 2 presenta los rangos de los criterios, éstos son la entrada al proceso de simulación con SMC. Los criterios $\mathrm{C}_{6}, \mathrm{C}_{7}$ y $\mathrm{C}_{2}$ se calcularon con las especificaciones del escenario base. Los cálculos de $\left(\mathrm{C}_{2}\right)$ se realizaron de acuerdo con la disponibilidad de la fuente y el área disponible en los parqueaderos. Para la FV se consideró: $2.512 \mathrm{~m}^{2}$, que corresponden al 95\% del área de techos de parqueo; la capacidad es de 1.500 paneles solares policristalinos de silicio de $2.600 \mathrm{Wp}$ de 1,66 $\mathrm{m} \times 0,99 \mathrm{~m}$. La radiación se modela con una función de distribución normal, con media $(\mu=5,625)$ y desviación estándar $(\sigma=0,41)$; los resultados de la modelación de la radiación y las características técnicas del sistema FV son las entradas para el proceso de simulación de la generación con SMC.

Para las turbinas eólicas se consideró el área total del parqueadero $\left(3.073 \mathrm{~m}^{2}\right)$, en el que es posible instalar 8 turbinas con las siguientes especificaciones: diámetro $5 \mathrm{~m}$, potencia nominal $5 \mathrm{~kW}$ (a $17 \mathrm{~m} / \mathrm{s}$ ), velocidad para arranque $2,5 \mathrm{~m} / \mathrm{s}$ y, altura $18 \mathrm{~m}$. La generación eólica diaria se determina a partir de la velocidad del viento con una función de distribución Weibull; la cual ya ha sido utilizada entre otros por Araujo et.al., (2009) quienes simularon la producción de aerogeneradores de pequeño tamaño. A partir de los datos presentados por Benavides y Ayala, (2010) se determinaron las constantes de escala $(c=5,2831)$ y de forma $(k=2,9639)$; con estos parámetros se realizó la simulación de la velocidad del viento $y$, junto con los datos técnicos de los aerogeneradores se simula la generación de la turbina eólica utilizando SMC. Los rangos de los otros criterios $\left(\mathrm{C}_{1}, \mathrm{C}_{3}, \mathrm{C}_{4}\right.$ y $\left.\mathrm{C}_{5}\right)$ se obtuvieron de la literatura sobre el tema. Para $\mathrm{C}_{6}$ y $\mathrm{C}_{7}$ no se presentan valores en la biomasa $(B)$, debido a que a través de los cálculos se determinó que no es suficiente para la generación en lo CCs.

Ranking de las alternativas (método TOPSIS). Los rangos de cada alternativa se simularon con SMC, utilizando una distribución uniforme de acuerdo con los propuesto Hadian y Madani, (2015). Se corrieron 100.000 simulaciones, empleando el software RISK5.5. Realizada la simulación, se escogió el valor que presenta mayor frecuencia y se procedió a unificar la escala de los valores aplicando una escala de 1 a 10 (10 es el mejor desempeño y 1 el menor). En la Fig. 8 se presentan los resultados del desempeño de cada criterio; el área cubierta por la línea en el radar muestra un alto (mayor área) o un bajo desempeño (menor área) de la alternativa. El ranking que se obtuvo se presenta en la 
Tabla 3. Es evidente la superioridad del desempeño de las FV frente a las otras alternativas.

Tabla 2. Criterios para la selección de las alternativas FER

\begin{tabular}{|c|c|c|c|c|}
\hline \multirow[t]{2}{*}{ Criterios } & \multirow[t]{2}{*}{ FER } & \multicolumn{2}{|c|}{ Valores } & \multirow[t]{2}{*}{ Referencias. } \\
\hline & & Min & $\operatorname{Max}$ & \\
\hline \multirow{3}{*}{$\begin{array}{l}C_{1} \text {. Uso de espacio: área total que ocupa } \\
\text { cada tecnología en el lugar en que es } \\
\text { instalada }\left(\mathrm{m}^{2} / \mathrm{kW}\right) \text {. }\end{array}$} & B & 1.000 & 6.000 & \multirow[t]{3}{*}{ (Troldborg, Heslop, y Hough, 2014) } \\
\hline & $\mathrm{FV}$ & 10 & 500 & \\
\hline & $E$ & 10 & 1200 & \\
\hline \multirow{3}{*}{$\begin{array}{l}C_{2} \text {. Generación diaria }\left(\mathrm{kWh} / \mathrm{m}^{2}\right) \text { : A partir } \\
\text { de la disponibilidad de la fuente se } \\
\text { calcula la capacidad de generación en el } \\
\text { edificio. }\end{array}$} & $\mathrm{B}$ & 0,0 & 0,0 & \\
\hline & $\mathrm{FV}$ & 0,63 & 1,13 & (UPME-IDEAM, 2005) \\
\hline & $E$ & 0,00 & 1,59 & (Benavides y Ayala, 2010) \\
\hline \multirow{3}{*}{$\begin{array}{l}C_{3} \text {. Emisiones: cantidad de dióxido de } \\
\text { carbono equivalente }\left(\mathrm{CO}_{2 e q}\right) \text { que se ha } \\
\text { estimado emite cada tecnología. }\end{array}$} & $B$ & 16,0 & 74,0 & \multirow[t]{3}{*}{ (NREL, 2014) } \\
\hline & $\mathrm{FV}$ & 39,0 & 49,0 & \\
\hline & $E$ & 3,0 & 45,0 & \\
\hline \multirow{3}{*}{$\begin{array}{l}\text { C. Eficiencia: mide la capacidad de } \\
\text { conversión de la fuente en electricidad } \\
(\%) \text {. }\end{array}$} & $B$ & 25,0 & 35,0 & (REN21, 2014) \\
\hline & $\mathrm{FV}$ & 10,0 & 30,0 & (NREL, 2015) \\
\hline & $E$ & 25,0 & 40,0 & (Evans, Strezov, y Evans, 2009) \\
\hline \multirow{3}{*}{$\begin{array}{l}C_{5} . \text { Madurez de la tecnología: se } \\
\text { determina la tasa de aprendizaje (\%). }\end{array}$} & $B$ & 2,1 & 9,0 & \multirow{3}{*}{$\begin{array}{l}\text { (Criqui, Mima, Menanteau, } \\
\text { Kitous, 2014), (Neij, 2008) y } \\
\text { (OCDE-AIE, 2012) }\end{array}$} \\
\hline & $\mathrm{FV}$ & 15,5 & 47,0 & \\
\hline & $E$ & 6,5 & 8,0 & \\
\hline \multirow{3}{*}{$\begin{array}{l}C_{6} \text {. CNE: costo de generación de un } \\
\text { kWh del sistema }(\$ / k W h)\end{array}$} & B & - & - & \multirow[t]{3}{*}{ De autores } \\
\hline & $\mathrm{FV}$ & 0,078 & 0,097 & \\
\hline & $\mathrm{E}$ & 0,46 & 3,28 & \\
\hline \multirow{3}{*}{$\begin{array}{l}C_{7} \text {. VPN: valor de los flujos del proyecto } \\
\text { en el año cero (US\$) }\end{array}$} & $B$ & - & - & \multirow[t]{3}{*}{ De autores } \\
\hline & $\mathrm{FV}$ & 75.428 & 154.884 & \\
\hline & E & -131.583 & -107.452 & \\
\hline
\end{tabular}
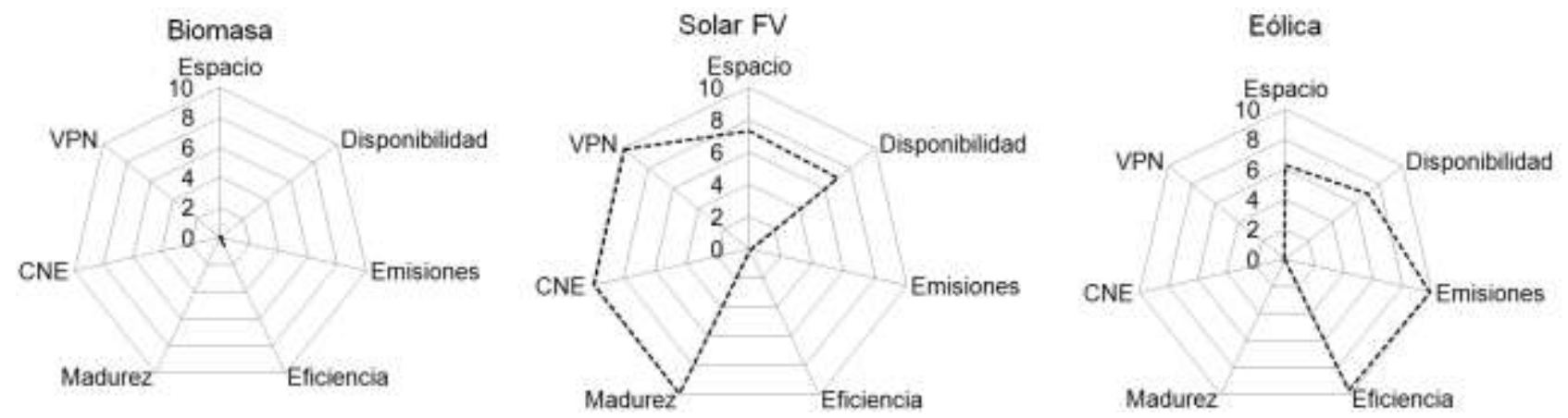

Fig. 8. Desempeño de los criterios para cada una de las alternativas

Tabla 3. Ranking de las alternativas para las dos estrategias

\begin{tabular}{lcc}
\hline \multicolumn{1}{c}{ Alternativas } & valor & Ranking \\
\hline FV & 0,749632 & 1 \\
Eólica & 0,316298 & 2 \\
Biomasa & 0,059688 & 3 \\
\hline
\end{tabular}


Evaluación económica del proyecto. Con las dos mejores alternativas seleccionadas en la etapa anterior, se estructuró arbitrariamente un portafolio (P1), compuesto por 750 paneles FV y 4 turbinas eólicas, que representan el $50 \%$ de la capacidad de instalación del CC. El P1 se evaluó con el método FDC incluyendo los incentivos de la L1715. Se realizaron 10.000 simulaciones y se obtuvieron los datos que se presentan en la Tabla 4, resultó: 1) el valor actual del portafolio (VA) presenta valores positivos, 2) el VPN promedio es negativo y su probabilidad de ser positivo es $2,9 \%$, 3) el CNE presenta un valor medio de 0,12 , valor que está por debajo del $\overline{\mathrm{PC}}$ de los $\mathrm{CC}$ de referencia y por encima de la media mundial. En la Fig 9 se observa la distribución del VPN y del VA del proyecto, como se puede observar la probabilidad de obtener valores positivos en el VPN (área a la derecha de cero, en el eje x) es muy baja; situación que desalentaría la inversión en el proyecto; se indica el valor de la inversión inicial (lo), los valores a la derecha de éste superan el desembolso inicial y son los que el inversionista esperaría.

Tabla 4. Estadísticos del Portafolio Uno

\begin{tabular}{|l|c|c|c|}
\hline Nombre & CNE & $V A$ & $V P N$ \\
\hline Mínimo (\$) & 0,08 & 423.428 & $(74.438)$ \\
\hline Máximo(\$) & 0,13 & 570.262 & 61.975 \\
\hline Media (\$) & 0,11 & 473.796 & -29.362 \\
\hline Desviación Std.(\$) & 0,01 & 15.129 & 14.464 \\
\hline Simetría & $(0,27)$ & 0,42 & 0,46 \\
\hline Curtósis & 3,12 & 3,69 & 3,86 \\
\hline Percentil 5\% (\$) & 0,10 & 450.697 & $(51.549)$ \\
\hline
\end{tabular}

La valoración de las OR se realiza bajo el supuesto de una ampliación del $50 \%$ de la capacidad instalada con respecto al portafolio uno, ésto es: paneles FV 1.125 y turbinas eólicas 8; las demás variables se mantienen iguales. En la Tabla 5 se relacionan los resultados de las dos opciones (columna: "No optimizadas"), las dos OR presentan incrementos considerables del valor actual $(\Delta V A)$ frente al portafolio uno, siendo la ORe de mayor crecimiento con un $707 \%$.

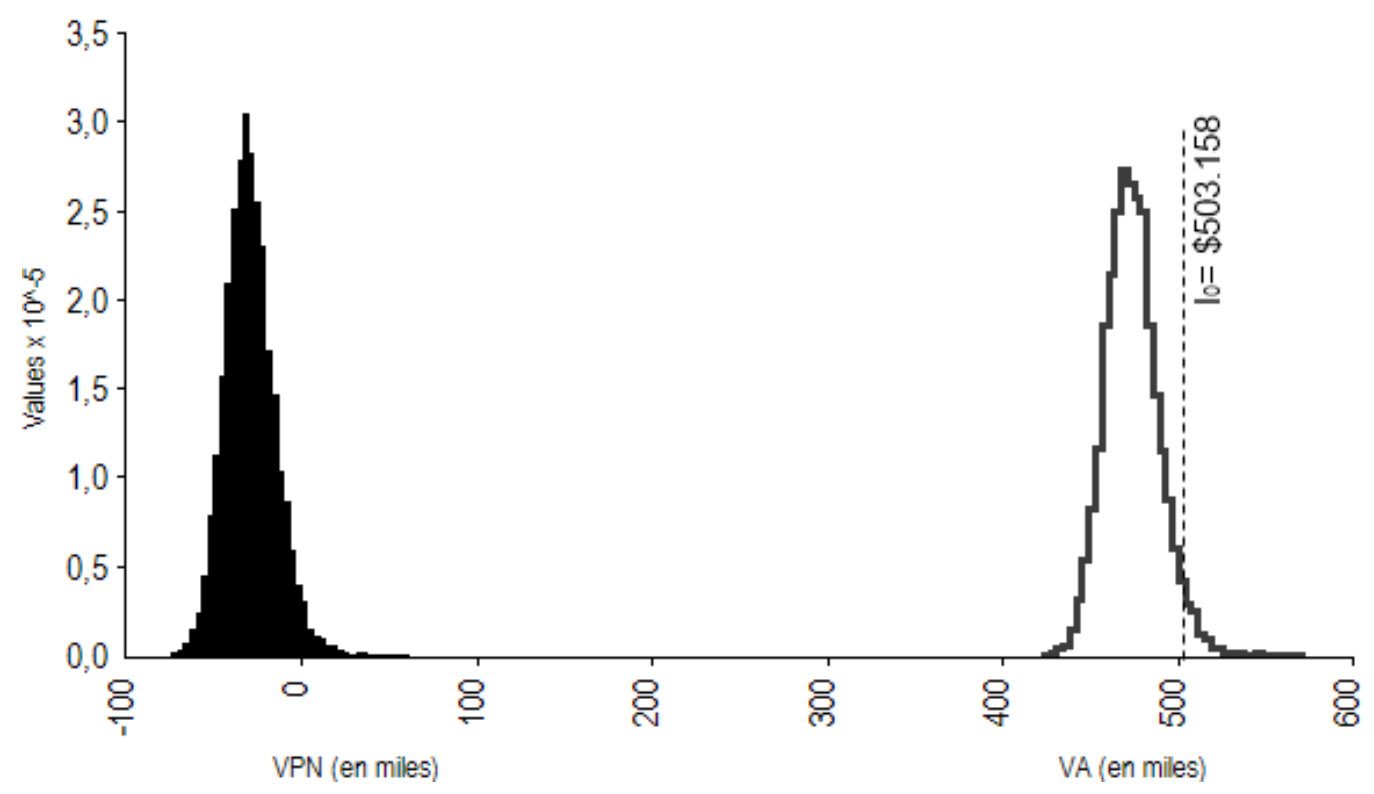

Fig. 9. Distribución del VPN y VA del proyecto.

Optimización del Portafolio FER. El proceso de optimización toma como referencia el portafolio uno, y se basó en las ecuaciones (14) y (15), se simulan dos casos: optimización 1) se realiza con restricciones de instalación mínima $\left(C_{F V}^{\text {actual }}=750\right.$ y $\left.C_{E}^{\text {actual }}=4\right)$ y máxima $\left(C_{F V}^{\max }=1500\right.$ y $\left.C_{E}^{\max }=8\right)$, optimización 2) a diferencia de la anterior la capacidad actual es cero. 
Tabla 5. Valor de las opciones optimizadas y no optimizadas.

\begin{tabular}{|l|c|c|c|c|c|c|}
\hline & \multicolumn{2}{|c|}{ No optimizadas } & \multicolumn{2}{c|}{ Optimización 1 } & \multicolumn{2}{c|}{ Optimización 2 } \\
\hline & $O R_{e}$ & $O R_{R D}$ & $O R_{e}$ & $O R_{R D}$ & $O R_{e}$ & $O R_{R D}$ \\
\hline Io adicional (\$) & 253.693 & 250.073 & 442.976 & 246.695 & 512.643 & 125.416 \\
\hline Beneficio adicional (\$) & 442.976 & 303.229 & 193.807 & 300.463 & 442.976 & 208.592 \\
\hline Paneles FV (und) & 1.125 & 1125 & 898 & 1117 & 1500 & 1014 \\
\hline Turbinas Eólicas (und) & 6 & 6 & 7 & 6 & 8 & 4 \\
\hline$\overline{V A}_{\mathrm{f}}(\$)$ & 179.969 & 68.765 & 266.732 & 70.701 & 666.809 & 77.968 \\
\hline$\Delta \mathrm{VA}$ (frente a P1) (\%) & 707 & 332 & 1.000 & 339 & 2.350 & 363 \\
\hline
\end{tabular}

Los resultados del proceso de optimización del portafolio se presentan en la Tabla 5. Hay mejoras importantes en el $\overline{V A}_{f}$ de las dos opciones después del proceso de optimización, e incluso se disminuye el número de paneles FV. En la optimización 1 las eólicas permanecieron constantes para las ORRD. Es claro como el proceso de optimización permitió incrementar el valor actual del portafolio frente al portafolio uno y mejorar los resultados de la aplicación de las opciones reales frente al portafolio uno en $2.350 \%$ para $R_{e}$ y en $363 \%$ para ORRD.

\section{CONCLUSIONES}

El enfoque sistémico del método, permitió integrar diferentes técnicas para encontrar a partir de la caracterización de un consumidor comercial y la selección de las alternativas FER (a través de las técnicas AHP y TOPSIS), el VPN expandido del proyecto, que incluye las OR implicitas del marco regulatorio colombiano. Se demostró que el método es eficaz y fácil de aplicar ya que recurre a técnicas y herramientas software que son de conocimiento en el campo académico y empresarial. El mismo proceso se puede llevar a cabo para edificios industriales y residenciales en Colombia; en otros paises se puede aplicar la metodología pero se requiere diseñar el modelo ecónomico de acuerdo al marco normativo y costos propios.

La aplicación de la metodología por etapas, permitió verificar la contribución de cada una de las técnicas aplicadas: 1) la caracterización del inversionista es la base del proceso, sin ésta todo el trabajo sería especulativo; 2) los métodos MTDM fundamentados en procesos científicos, mejoran la selección de las alternativas, sin dejar de lado la incertidumbre del proceso de toma de decisiones. Este método permitió descartar la biomasa debido a su baja producción, como alternativa de generación en CC; 3) La TOR permitió valorar las OR del proyecto implicitas en la L1715; 4) la simulación y optimización del portafolio incluyendo las OR, además de generar mejores resultados, establecen una forma apropiada de evaluar este tipo de proyectos y; 5) considerar la incertidumbre en todos los procesos de cálculo, permitió obtener un espectro más amplio del comportamiento de las inversiones en el tiempo y mejorar la confiabilidad de los resultados.

La aplicación del MTDM con la participación de colombianos expertos en el sector de las renovables, permitió determinar después de los procesos de comparación de los criterios, que la disponibilidad de la fuente es el atributo más importante a considerar en los procesos de evaluación de altenativas FER para edificios comerciales en Colombia. Esta postura difiere de otros estudios en que las emisiones [ver IbáñezForés y Perez-Belis (2014)] y los costos de inversión [ver Jiang-Jiang, Chun-Fa, y Guo-Zhong (2009)] son los criterios que ocupan el primer lugar.

Se demostró que un proyecto que incialmente se consideraba inviable, a través de la evaluación de las OR y la optimización del portafolio puede convertirse en atractivo bajo las condiciones analizadas en el contexto del caso de estudio. De tal manera que una conclusión importante para el sector eléctrico y comercial en Colombia, es que el nuevo marco regulatorio y las condiciones metereológicas, que se caracterizan por altos niveles de radiación en mayor parte del territorio colombiano, son oportunidades propicias para promover la implementación de tecnologías FER y la participación en programas RD y venta de excedentes.

\section{REFERENCIAS}

Agencia Internacional de Energía. The Impact of Global Coal Supply on Worldwide Electricity Prices, https://www.iea.org/publications, 32, IEA/OECD , Paris, Francia, (2014) 
Alcan, P., Balin, A. y Baslıgil, H. Fuzzy multicriteria selection among cogeneration systems: A real case application, doi:10.1016/j.enbuild.2013.08.048, Energy and Buildings, 67, 624-634, (2013)

Aragonés-Beltrán, P., Chaparro-González, F., Pastor-Ferrando, J. y Pla-Rubio, A. An AHP (Analytic Hierarchy Process)/ANP (Analytic Network Process)-based multi-criteria decision approach for the selection of solar-thermal power plant investment projects, doi:10.1016/j.energy.2013.12.016, Energy, 66, 222-238, (2014)

Araujo, A. M., Melo, G. M., de Medeiros, A. y dos Santos, M. J. Simulación de la Producción de Energía Eléctrica con Aerogeneradores de Pequeño Tamaño, Información Tecnológica, doi:10.4067/S071807642009000300006, 20(3), 37-44, (2009)

Awerbuch, S. y Yang, S. Efficient Electricity Generating Portfolios for Europe: Maximizing Energy Security and Climate Change Mitigation. Analytical Methods for Energy Diversity and Security, 87-115, Oxford, UK, (2008)

Belderbos, A., y Delarue, E. Accounting for flexibility in power system planning with renewables, doi:10.1016/j.ijepes.2015.02.033, Electrical Power and Energy Systems, 71, 33-41, (2015)

Benavides, H. O., y Ayala, L. Análisis Descriptivo de Variables Metereológicas que Influyen en la Calidad del Aire de los Principales Centros Industriales del País, IDEAM, https://www.siac.gov.co/documentos/DOC_Portal/DOC_Clima/070912_NT_Anal_VarMeteo_Caire_CIInd_pai s.pdf, Bogotá (2010)

Bhattacharya, A. y Kojima, S. Power sector investment risk and renewable energy: A Japanese case study using portfolio risk optimization method, doi:10.1016/j.enpol.2010.09.031, Energy Policy, 40, 69-80, (2012)

Ceciliano, J. L., Bayram, M. y Masud, A. A Model for the Multiperiod Multiobjective Power Generation Expansion Problem, doi:10.1109/TPWRS.2007.895178, IEEE TRANSACTIONS ON POWER SYSTEMS, 22(2), 871-879, (2007)

Cervi, R. G., Esperancini, M. S. y Bueno, O. Viabilidad Económica de la Utilización de Biogás para la Conversión en Energía Eléctrica, doi:10.4067/S0718-07642011000400002, Información Tecnológica, 22(4), 3-14, (2011)

Congreso de la República de Colombia. Ley $1715 \quad$ de 2014 , http://www.secretariasenado.gov.co/senado/basedoc/ley_1715_2014.html, Bogotá, (2014)

Copeland, T. y Antikarov, V. Real options: A Practitioner`s guide. ISBN-10: 1587991861, United Kingdom: Penguin Group, (2001)

Cox, J., Ross, S. y Rubinstein, M. Options Pricing: A simplified approach, doi:10.1016/0304-405X(79)900151, Journal of Financial Economics, 7, 229-263, (1979)

Criqui, P., Mima, S., Menanteau, P. y Kitous, A. Mitigation strategies and energy technology learning: An assessment with the POLES model, doi:10.1016/j.techfore.2014.05.005, Technological Forecasting, 90, 118, (2014)

Delarue, E., De Jonghe, C., Belmans, R. y D'Haeseleer, W. Ceciliano, doi:10.1016/j.eneco.2010.05.003, Energy Economics, 33, 12-23. (2011)

Departamento de Energía de E.U. Benefits of Demand Response in Electricity Markets and Recomendations for Achieving them, http://energy.gov/sites/prod/files/oeprod/DocumentsandMedia/DOE_Benefits_of_Demand_Response_in_Ele ctricity_Markets_and_Recommendations_for_Achieving_Them_Report_to_Congress.pdf, (2006)

Evans, A., Strezov, V. y Evans, T. J. Assessment of sustainability indicators for renewable energy technologies, doi:10.1016/j.rser.2008.03.008, Renewable and Sustainable Energy Reviews, 13(2), 10821088, (2009)

Fuss, S., Szolgayova, J., Khabarov, N., y Obersteiner, M. Renewables and climate change mitigation: Irreversible energy investment under uncertainty and portfolio effects, doi:10.1016/j.enpol.2010.06.061, Energy Policy, 40, 59-68, (2012) 
Hadian, S. y Madani, K. A system of systems approach to energy sustainability assessment: Are all renewables really green?, doi:10.1016/j.ecolind.2014.11.029, Ecological Indicators, 52, 194-206, (2015)

Ibáñez-Forés, V. y Perez-Belis, V. A holistic review of applied methodologies for assessing and selecting the optimal technological alternative from a sustainability perspective, doi:10.1016/j.jclepro.2014.01.082, Journal of Cleaner Production, 70, 1-23, (2014)

Jiang-Jiang, W., Chun-Fa, Z. Y.-Y. y Guo-Zhong, Z. Review on Multicriteria decision analysis and in sustainable energy decision-making, doi:10.1016/j.rser.2009.06.021, Renewable and Sustainable Energy Reviews, 13(9), 2263-2278, (2009)

Kienzle, F., Ahčin, P. y Göran, A. Valuing Investments in Multi-Energy Conversion, Storage, and DemandSide Management Systems Under Uncertainty, doi:10.1109/TSTE.2011.2106228, IEEE TRANSACTIONS ON SUSTAINABLE ENERGY, 2(2), 194-202, (2012)

Lee, S.-C. Using real option analysis for highly uncertain technology investments: The case of wind energy technology, doi:10.1016/j.rser.2011.07.107, Renewable and Sustainable Energy Reviews, 15(9), 4443-4450, (2011)

Loken, E. y Botterud, A. Planning of Mixed Local Energy Distributions Systems: A comparison of two multicriteria decision methods, Sustainable energy dsitribution Systemns: Planning Methods and Models, SINTEF Energy Research, Trondhein, Noruega, (2005)

Manotas-Duque, D. F., Oliveros, D. A., Taborda, H. F., Vidal-Holguín, C. J. y Lozano, C. A. Energy supply optimization for unregulated consumers, http://www.scielo.org.co/pdf/inco/v16n1/v16n1a09.pdf, ISSN 01233033, Ingeniería y Competitividad, 16(1), 97-107, (2014)

Martinez-Cesena, E., Azzopardi, B. y Mutale, J. Assessment of domestic photovoltaic systems based on real options theory, doi:10.1002/pip.2208, Progress in Photovoltaics: Research and Applications, 21(2), 250-262, (2013)

Neij, L. Cost development of future technologies for power generation-A study based on experience curves and complementary bottom-up assessments. doi:10.1016/j.enpol.2008.02.029, Energy Policy, 36(6), 1-12, (2008).

NREL. Life Cycle Assessment Harmonization Results and Findings. http://www.nrel.gov/analysis/sustain_lca_results.html, acceso el 14 de Abril de 2015 de National Renewable Energy Laboratory, (2014)

NREL. National Renewable Electricity Laboratory. acceso el 21 de 4 de 2015, de http://www.nrel.gov/ncpv/images/efficiency_chart.jpg, (2015)

OCDE-AIE. Energy Technology Perspectives 2012. Pathways to a clean Energy System, ISBN: 978-92-6417488-7, 59-107, OCDE/AIE, Paris, Francia, (2012)

REN21. Renewables 2014, GLOBAL STATUS REPORT. Worldwatch Institute, ISBN 978-3-9815934-2-6, 48-58, REN 21, Paris, Francia, (2014)

Saaty, T. y Vargas, L. G. (2001). Models, Methods, Concepts y Applications of the Analytic Hierarchy Process, 1 ed, pp 6, Springer Science, New York, Estados Unidos, (2001)

San Cristobal M, J. R. Multi-criteria decision-making in the selection of a renewable energy project in spain: The Vikor method, doi:10.1016/j.renene.2010.07.031, Renewable Energy, 36(2), 498-502, (2011)

Santos, L., Soares, I., Mendes, C. y Ferreira, P. Real Options versus Traditional Methods to assess Renewable Energy Projects, 10.1016/j.renene.2014.01.038, Renewable Energy, 68, 588-594, (2014)

Sezgen, O. y Krishnarao, G. Option value of electricity demand response,doi:10.1016/j.energy.2006.03.024, Energy, 32(2), 108-119, (2007)

Spronk, J. y Hallerbach, W. Financial modelling: Where to go? With an illustration for portfolio management, doi:0377-2217(96)003 86-4, European Journal of Operational Research, 99, 113-125, (1997) 
Stirling, A. Multicriteria diversity analysis A novel heuristic framework for appraising energy portfolios, 10.1016/j.enpol.2009.02.023, Energy Policy, 38, 1622-1634, (2010)

Taha, R. A. y Daim, T. Multi-Criteria Applications in Renewable Energy Analysis, a Literature Review. Research and Technology Management in the Electricity Industry: Methods, Tools and Case Studies, ISBN 978-1-4471-5097-8, Springer-Verlag, pp. 17-30, Londres, Inglaterra, (2013)

Troldborg, M., Heslop, S. y Hough, R. L. Assessing the sustainability of renewable energy technologies using multi-criteria analysis:Suitability of approach for national-scale assessments and associated uncertainties, doi:10.1016/j.rser.2014.07.160, Renewable and Sustainable Energy Reviews, 39, 1173-1184, (2014)

UPME-CORPOEMA. Determinación del Potencial de Reducción del Consumo Energético en el Sector Servicios en Colombia, Ministerio de Minas y Energía, Bogotá, (2013)

UPME-IDEAM. Atlas de Radiación Solar de Colombia, Ministerio de Minas y Energía-Colombia, Bogotá, (2005)

Wang, J.-J., Jing, Y.-Y., Zhang, C.-F., Shi, G.-H. y Zhang, X.-T. A fuzzy multi-criteria decision-making model for trigeneration system, doi:10.1016/j.enpol.2008.07.002, Energy Policy, 36, 3823-3832, (2008) 